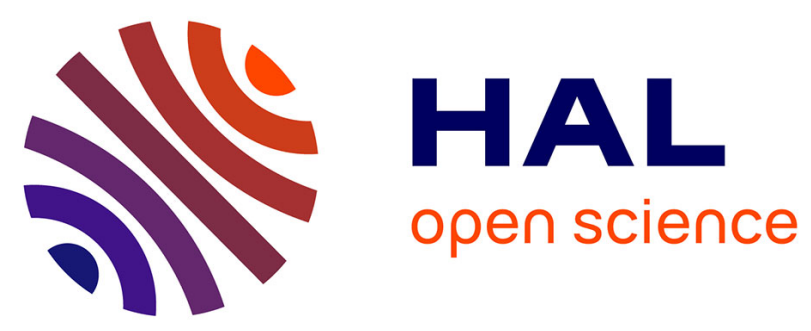

\title{
Spectrum Sensing in Cognitive Radio Networks
}

Leonardo Sampaio Cardoso, Merouane Debbah, Samson Lasaulce, Kobayashi

\author{
Mari, Jacques Palicot
}

\section{To cite this version:}

Leonardo Sampaio Cardoso, Merouane Debbah, Samson Lasaulce, Kobayashi Mari, Jacques Palicot. Spectrum Sensing in Cognitive Radio Networks. Cognitive Radio Networks: Architectures, Protocols and Standards, CRC Press, pp.3-28, 2010, 10.1201/ebk1420077759-c1 . hal-00448800

\section{HAL Id: hal-00448800 \\ https://hal-centralesupelec.archives-ouvertes.fr/hal-00448800}

Submitted on 20 Jan 2010

HAL is a multi-disciplinary open access archive for the deposit and dissemination of scientific research documents, whether they are published or not. The documents may come from teaching and research institutions in France or abroad, or from public or private research centers.
L'archive ouverte pluridisciplinaire HAL, est destinée au dépôt et à la diffusion de documents scientifiques de niveau recherche, publiés ou non, émanant des établissements d'enseignement et de recherche français ou étrangers, des laboratoires publics ou privés. 
Book Title: Cognitive Radio Networks

Editors

November 5, 2008 


\section{Contents}

1 Spectrum Sensing in Cognitive Radio Networks 1

1.1 Introduction . . . . . . . . . . . . . . . . . . 2

1.1.1 Interference Management and Spectrum Sensing . . . . . . . . . . . 2

1.1.2 Characteristics of Spectrum Sensing . . . . . . . . . . . 3

1.2 Problem Formulation . . . . . . . . . . . . . . . . . . 4

1.2.1 The General Spectrum Sensing Problem . . . . . . . . . . . . . . 4

1.2.2 Spectrum Sensing from the Cognitive Radio Network Perspective . . 7

1.3 Non-Cooperative Sensing Techniques . . . . . . . . . . . . . . . . . 8

1.3.1 Energy Detector . . . . . . . . . . . . . . . . 9

1.3.2 Matched Filter Detector . . . . . . . . . . . . . . . . 12

1.3.3 Cyclostationary Feature Detection . . . . . . . . . . . 15

1.4 Cooperative Sensing Techniques . . . . . . . . . . . . . . . 16

1.4.1 Voting Based Sensing . . . . . . . . . . . . . . . . . . 19

1.4.2 Correlator Based Sensing . . . . . . . . . . . . . . . . . 21 
1.4.3 Eigenvalue Based Sensing _. . . . . . . . . . . . . . . . . . 23

1.5 Conclusions and Open Issues . . . . . . . . . . . . . . . . . . . . 27 


\section{Chapter 1}

\section{Spectrum Sensing in Cognitive Radio}

\section{Networks}

Leonardo S. Cardoso, Mérouane Debbah, Samson Lasaulce, Mari Kobayashi and Jacques Palicot

Today, the creation of new radio access technologies is limited by the shortage of the available radio spectrum. These new technologies are becoming evermore bandwidth demanding due to their higher rate requirements. Cognitive radio networks and spectrum sensing techniques are a natural way to allow these new technologies to be deployed.

In this chapter we will discuss spectrum sensing for cognitive radio networks. We will begin by introducing the subject in Sec. 1.1, providing a brief background followed by a discussion of spectrum sensing motivations and characteristics. Then we will move on to the spectrum sensing problem itself in Sec. 1.2, where we explain the issues that are inherent to spectrum sensing. In Sec. 1.3 we will explore the classical non-cooperative spectrum sensing techniques which form the basis for the more elaborate, cooperative techniques presented in Sec. 1.4. Finally we will close this chapter with some conclusions and open issues. 


\section{$1.1 \quad$ Introduction}

One of the most prominent features of cognitive radio networks will be the ability to switch between radio access technologies, transmitting in different portions of the radio spectrum as unused frequency band slots arise $[2,3,4]$. This dynamic spectrum access is one of the fundamental requirements for transmitters to adapt to varying channel quality, network congestion, interference and service requirements. Cognitive radio networks (from now on called secondary networks) will also need to coexist with legacy ones (hereafter called primary networks), which have the right to their spectrum slice and thus can not accept interference.

Based on these facts, under-utilization of the current spectrum and the need to increase the network capacity is pushing research towards new means of exploiting the wireless medium. In this direction, the Federal Communications Commission (FCC) Spectrum Policy Task Force has published a report [5] in 2002, in which it thoroughly investigates the under utilization of the radio spectrum. While the FCC is in charge of determining the spectrum usage and its policies, the Whitespace Coalition, formed by companies such as Microsoft, Google, Dell, HP and Intel is studying ways to exploit the spectrum vacancies in the television band. Cognitive radio networks are envisioned to be able to opportunistically exploit those spectrum "left-overs", by means of knowledge of the environment and cognition capability, in order to adapt their radio parameters accordingly. Spectrum sensing is the technique that will enable cognitive radio networks to achieve this goal.

\subsubsection{Interference Management and Spectrum Sensing}

In order to share the spectrum with legacy systems, cognitive radio networks will have to respect some set of policies, defined by regulatory agencies $[3,4]$. These policies are based on the central idea where there are primary systems which have the right to the spectrum and secondary systems which are allowed to use the spectrum so long as they do not disturb the communications of the primary systems. Roughly speaking, these policies deal with controlling the amount of interference that the secondary systems can incur to primary ones. Thus, the problem is one of interference management $[3,4]$. We can address this problem 
from two different points of view: receiver centric or transmitter centric.

\section{Receiver Centric Interference Management}

In the receiver centric approach $[3,4]$, an interference limit at the receiver is calculated and used to determine the restriction on the power of the transmitters around it. This interference limit, called the interference temperature, is chosen to be the worst interference level that can be accepted without disturbing the receiver operation beyond its operating point. Although very interesting, this approach requires knowledge of the interference limits of all receivers in a primary system. Such knowledge depends on a many variables, including individual locations, fading situations, modulations, coding schemes and services. Receiver centric interference management techniques will not be addressed in this chapter as they have been recently ruled out by the IEEE SCC41 cognitive radio network standard.

\section{Transmitter Centric Interference Management}

In the transmitter centric approach, the focus is shifted to the source of interference $[3,4]$. The transmitter does not know the interference temperature, but by means fo sensing, it tries to detect free bandwidth. The sensing procedure allows the transmitter to classify the channel status to decide whether it can transmit and with how much power. In actual systems, however, since the transmitter does not know the location of the receivers or their channel conditions, it is not able to infer how much interference these receivers can tolerate. Thus, spectrum sensing solves the problem for worst case scenario, assuming strong interference channels, so the secondary system transmits only when it senses an empty medium.

\subsubsection{Characteristics of Spectrum Sensing}

There are several techniques available for spectrum sensing, each with its own set of advantages and disadvantages that depends on the specific scenario. Some works in the literature $[6,7,8]$ consider spectrum sensing as a method for distinguishing between two or more 
different types of signals or technologies in operation. Since this is not a question of detection (determining whether a given frequency band is being used), these types of signal identification issues such as [9] are not addressed in this chapter. Rather we focus on their detection.

Ultimately, a spectrum sensing device must be able to give a general picture of the medium over the entire radio spectrum. This allows the cognitive radio network to analyze all degrees of freedom (time, frequency and space) in order to predict the spectrum usage. Wideband spectrum sensing works are also available in the literature [10, 11, 12, 13], however, equipment able to perform wide-band sensing all at once is prohibitively difficult to build with today's technology. Feasible spectrum sensing device can quickly sweep the radio spectrum, analyzing one narrowband segment at a time. This chapter will focus on narrowband sensing techniques.

In this section, we have emphasized the importance of the spectrum sensing technique for cognitive radio networks. In the next section, we aim at understanding the underlying characteristics of the spectrum sensing problem, which will enable us to develop the approaches presented further in this chapter.

\subsection{Problem Formulation}

\subsubsection{The General Spectrum Sensing Problem}

Spectrum sensing is based on a well known technique called signal detection. In a nutshell, signal detection can be described as a method for identifying the presence of a signal in a noisy environment. Signal detection has been throughly studied for radar purposes since the fifties [1]. Analytically, signal detection can be reduced to a simple identification problem, formalized as a hypothesis test $[14,15,16]$ : 


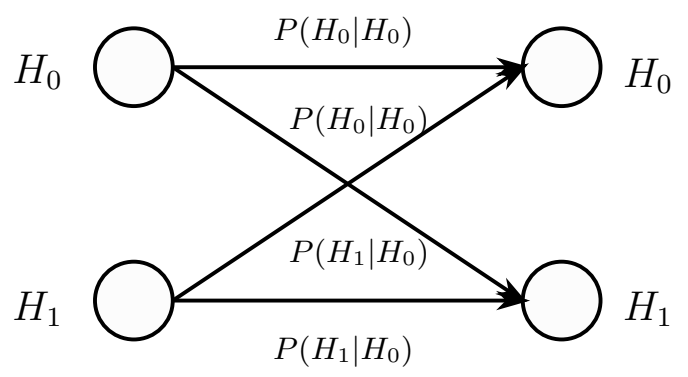

Figure 1.1: Hypothesis test and possible outcomes with their corresponding probabilities.

$$
y(k)=\left\{\begin{array}{ll}
n(k): & H_{0} \\
s(k)+n(k): & H_{1}
\end{array},\right.
$$

where $y(k)$ is the sample to be analyzed at each instant $k, n(k)$ is noise (not necessarily white Gaussian noise) of variance $\sigma^{2}, s(k)$ is the signal the network wants to detect, and $H_{0}$ and $H_{1}$ are the noise-only and signal plus noise hypotheses, respectively.

$H_{0}$ and $H_{1}$ are the sensed states for absence and presence of signal, respectively. Then, as seen in Fig. 1.1 we can define four possible cases for the detected signal:

1. declaring $H_{0}$ when $H_{0}$ is true $\left(H_{0} \mid H_{0}\right)$;

2. declaring $H_{1}$ when $H_{1}$ is true $\left(H_{1} \mid H_{1}\right)$;

3. declaring $H_{0}$ when $H_{1}$ is true $\left(H_{0} \mid H_{1}\right)$;

4. declaring $H_{1}$ when $H_{0}$ is true $\left(H_{1} \mid H_{0}\right)$.

Case 2 is known as a correct detection, whereas cases 3 and 4 are known as a missed detection and a false alarm, respectively. Clearly, the aim of the signal detector is to achieve correct detection all of the time, but this can never be perfectly achieved in practice because of the statistical nature of the problem. Therefore, signal detectors are designed to operate within prescribed minimum error levels. Missed detections are the biggest issue for spectrum sensing, as it means possibly interfering with the primary system. Nevertheless, it is desirable to keep the false alarm rate as low as possible for spectrum sensing, so that the system can exploit all possible transmission opportunities. 
The performance of the spectrum sensing technique is usually influenced by the probability of false alarm $P_{f}=P\left(H_{1} \mid H_{0}\right)$, since this is the most influential metric. Usually, the performance is presented by receiver operation characteristics (ROC) curves, which plot the probability of detection $P_{d}=P\left(H_{1} \mid H_{1}\right)$ as a function of the probability of false alarm $P_{f}$.

Equation (1.1) shows that, to distinguish $H_{0}$ and $H_{1}$, a reliable way to differentiate signal from noise is required. This becomes very difficult in the case where the statistics of the noise are not well known or when the signal to noise ratio (SNR) is low, in which case the signal characteristics are buried under the noise, as shown by Tandra et. al. in [17]. In fact, this work also shows that the less one knows about the statistics of the noise, the worse the performance of any signal detector is in the low SNR regime.

Clearly, the noise characteristics are very important for the spectrum sensing procedure. Most works on spectrum sensing consider noise to be AWGN, since many independent sources of noise are added (central limit theory). Nevertheless, in realistic scenarios, this approximation may not be appropriate since receivers modify the noise through processes such as filters, amplifier non-linearities and automatic gain controls [18, 19].

Poor performance in a low SNR regime means that all of the techniques available are negatively affected by poor channels. In the case of variable channel gains, Eq. 1.1 is rewritten as:

$$
y(k)= \begin{cases}n(k): & H_{0} \\ h(k) s(k)+n(k): & H_{1}\end{cases}
$$

where $\mathrm{h}(\mathrm{k})$ is the channel gain at each instant $k$. In a wireless radio network, since it is reasonable to assume that the spectrum sensing device does not know the location of the transmitter, two options arise:

- A low $h(k)$ is solely due to the pathloss (distance) between the transmitter and the sensing device meaning that the later is out of range and can safely transmit;

- A low $h(k)$ is due to shadowing or multipath, meaning that the sensing device might 
be within the range of the transmitter and can cause harmful interference.

In the later case, a critical issue arises. Therein, the fading plays an especially negative role in the well known "hidden node" problem [20]. In this problem, the spectrum sensing terminal is deeply faded with respect to the transmitting node while having a good channel to the receiving node. The spectrum sensing node then senses a free medium and initiates its transmission, which produces interference on the primary transmission. Thus, fading here introduces uncertainty regarding the estimation problem. To solve this issue, cooperative sensing has been proposed. In this approach, several sensing terminals gather their information in order to make a joint decision about the medium availability. Cooperative spectrum sensing will be further explored in Sec. 1.4.

\subsubsection{Spectrum Sensing from the Cognitive Radio Network Perspective}

In contrast with the general case, where only the signal detection aspect is considered, the problem of spectrum sensing as seen from a cognitive radio perspective has very stringent restrictions. These are mainly imposed by the policies these cognitive radio networks face in order to be able to operate alongside legacy networks. Some of these restrictions are summarized below:

\section{No prior knowledge on the signal structure}

There are portions of the spectrum where multiple technologies (using different protocols) share the spectrum, such as the ones operating on the instrumentation scientific and medical (ISM) unlicensed band. Cognitive radio networks must be able to deal with existing multiple technologies, as well as new ones that may eventually appear across the span of the wireless radio spectrum. These networks should be able to discover the state of the medium irrespective of the technologies in use. Of course, if the technologies are known, then this information can be exploited to improve the accuracy of the spectrum sensing, for example through the detection of known pilot sequences within the signal [17]. 


\section{Sensing Time}

Due to the primary importance of the legacy system, the secondary system must be designed to free the medium as soon as it senses that a legacy network has initiated a transmission. For efficient use of the spectrum, these secondary networks must also sense available spectrum as quickly as possible, in the least possible number of received samples. In general terms, spectrum sensing techniques work through a compromise between the number of samples and accuracy. Cooperative spectrum sensing gives the opportunity to decrease the sensing time for the same level of accuracy.

\section{Fading Channels}

As discussed earlier, spectrum sensing is particularly sensitive to fading environments. Communication systems operate in diverse environments, including those prone to fading. Thus, in many situations spectrum sensing devices must be able to detect reliably even over heavily faded channels. Although several works have focused in sensing for the fading environment in the non-cooperative setting $[21,22]$, it is foreseen that cooperative sensing $[32,33,34,42,44,35,36,37,41]$ is the best way to address this problem. Nevertheless, it creates other implications such as the distribution of metrics among the sensing terminals and the decision of which terminals are to be considered dependable or not.

\subsection{Non-Cooperative Sensing Techniques}

In a realistic spectrum sensing scenario there are situations in which only one sensing terminal is available or in which no cooperation is alowed due to the lack of communication between sensing terminals. In this section we will explore the main single user sensing schemes, some of which will serve as basis for the development of the cooperative ones, investigated in Sec. 1.4 .

Single user spectrum sensing approaches have been heavily studied in the literature, in 
part because of the relationship to signal detection. There are several classical techniques for this purpose, including the energy detector (ED)[16, 21, 22], the matched filter (MF)[23, 34] and the cyclostationary feature detection (CFD) [25, 26, 27, 28, 29].

\subsubsection{Energy Detector}

The most well known spectrum sensing technique is the energy detector. It is based on the principle that, at the reception, the energy of the signal to be detected is always higher than the energy of the noise. The energy detector is said to be a blind signal detector because it ignores the structure of the signal. It estimates the presence of a signal by comparing the energy received with a known threshold $\nu[16,21,22]$, derived from the statistics of the noise.

Let $y(k)$ be a sequence of received samples $k \in\{1,2, \ldots, N\}$ at the signal detector, such as in Eq. (1.1). Then, the decision rule can be stated as:

$$
\text { decide for }\left\{\begin{array}{ll}
H_{0}, & \text { if } \mathcal{E}<\nu \\
H_{1}, & \text { if } \mathcal{E} \geq \nu
\end{array}\right. \text {, }
$$

where $\mathcal{E}=E\left[|y(k)|^{2}\right]$ is the estimated energy of the received signal and $\nu$ is chosen to be the noise variance $\sigma^{2}$. In practice, one does not dispose of the actual received energy power $\mathcal{E}$. The energy detector uses, instead, the approximation $\hat{\mathcal{E}}$ for $\mathcal{E}$, where

$$
\hat{\mathcal{E}} \triangleq \frac{1}{N} \sum_{k=1}^{N}|y(k)|^{2}
$$

As the number of samples $N$ becomes large, then by the law of the large numbers $\hat{\mathcal{E}}$ converges to $\mathcal{E}$.

The energy detector is one of the simplest signal detectors. Its operation is very straightforward and it has a very easy implementation, since it depends only on simple and readily 
available information.

Nevertheless, in spite of its simplicity, the energy detector is not a perfect solution. The approximation of signal energy $\mathcal{E}$ gets better as $N$ increases. Thus, the performance of the energy detector is directly linked to the number of samples. Furthermore, the energy detector relies completely on the variance of the noise $\sigma^{2}$ which is taken as a fixed value. This is generally not true in practice, where the noise floor varies. Essentially this means that the energy detector will generate errors during those variations, specially when the SNR is very low, as seen in Figure 1.2(b), where we see an area of uncertainty surrounding the threshold $\nu$ in contrast with the case portrayed in Fig. 1.2(a), in which perfect noise knowledge is considered.

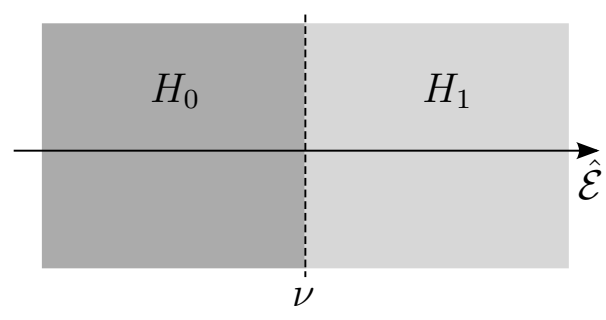

(a) Ideal energy detector scheme.

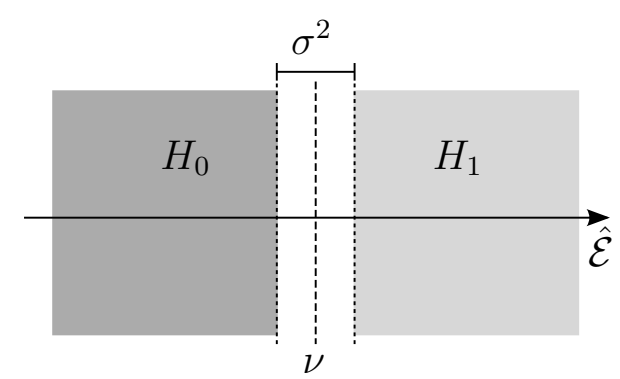

(b) Detection uncertainty for the energy detector

Figure 1.2: Ideal and actual energy detection schemes.

\section{Characterization of Energy Detector in AWGN Channels}

This case has been studied in the work of Urkowitz in 1967 [16]. It is known that the energy detection is the optimal signal detector in AWGN considering no prior information on the signal structure [17]. In order to understand the inner workings of the energy detector in this scenario, we need to understand how does the probability of detection $P_{d}=\operatorname{Prob}\left\{\hat{\mathcal{E}}>\nu \mid H_{1}\right\}$ and false alarm $P_{f}=\operatorname{Prob}\left\{\hat{\mathcal{E}}>\nu \mid H_{0}\right\}$ behaves with the measured received signal energy.

Take $n(k) \sim \mathcal{N C}\left(0, \sigma^{2}\right)$ as the AWGN noise sample. Then we know that for the noiseonly case, the distribution of the energy of $n$ over $T$ samples can be approximated by a zero mean chi-square distribution $\chi_{2 T W}^{2}[16]$, where $W$ is the total bandwidth. Similarly, the energy over $T$ samples of the sum of a signal plus noise, can be represented by a non-central 
chi-square distribution $\chi_{2 T W}^{2}(\lambda)[16]$, where $\lambda$ is the non-centrality parameter. Briefly:

$$
\hat{\mathcal{E}} \sim\left\{\begin{array}{ll}
\chi_{2 T W}^{2}, & H_{0} \\
\chi_{2 T W}^{2}(\lambda), & H_{1}
\end{array} .\right.
$$

With these considerations in mind, we can restate Eqs. ?? and ?? as:

$$
P_{f}=Q_{m}(\sqrt{\lambda \xi}, \sqrt{\nu})
$$

and

$$
P_{d}=\frac{\Gamma(T W, \nu / 2)}{\Gamma(T W)}
$$

where $Q_{m}$ is the Marcum Q-function, $\Gamma$ is the gamma function and $\xi$ is the signal to noise ratio seen by the signal detector.

\section{Characterization of Energy Detector in Fading Channels}

In 2002, Kostylev studied the performance of the energy detector in fading channels [21]. He derived analytical expressions for the energy detector over the Rayleigh fading channel case (also analyzed the Rice and Nakagami cases numerically). In 2003, the problem was revisited by Digham et. al. [22], who provided an alternative analytical development for these three kinds of fading channels. In this section, however, we will restrict the analysis to the more commonly adopted Rayleigh fading.

Let us begin by recalling that, in this case, the model focused is that shown in Eq. 1.14. As such, similar to what Urkowitz did in [16], Kostylev characterized the statistics of the energy of the signal for both the $H_{0}$ and $H_{1}$ cases, under the assumption that $h(k)$ is Rayleigh distributed: 


$$
\hat{\mathcal{E}} \sim \begin{cases}\chi_{2(T W+1)}^{2}, & H_{0} \\ e_{2\left(\xi^{2}+1\right)}+\chi_{2 T W}^{2}(\lambda), & H_{1}\end{cases}
$$

where $e_{2\left(d^{2}+1\right)}$ is the exponential distribution with parameter $\alpha=2\left(\xi^{2}+1\right)$ with probability density function $f(x, \alpha)=\alpha e^{-\alpha x}$ and $\xi$ is the signal to noise ratio.

It is clear to see that, under the hypothesis $H_{0}$, the statistics are the same as for the AWGN channel case, so the probability of false alarm is:

$$
P_{f}=Q_{m}(\sqrt{\lambda \xi}, \sqrt{\nu})
$$

However, the $H_{1}$ case behaves differently and has the probability of detection as given by $[22]:$

$$
P_{d}=e^{\hat{\frac{\mathcal{E}}{2}}} \sum_{m=0}^{T W-2} \frac{1}{m !}\left(\frac{\hat{\mathcal{E}}}{2}\right)+\left(\frac{1+\xi}{\xi}\right)^{T W-1}\left[e^{\frac{\hat{\mathcal{E}}}{2(1+\xi)}}-e^{\frac{\hat{\mathcal{E}}}{2}} \sum_{m=0}^{T W-2} \frac{1}{m !} \frac{\hat{\mathcal{E}} \xi}{2(1+\xi)}\right] .
$$

\subsubsection{Matched Filter Detector}

We have seen previously in Sec. 1.3.1 that the best sensing technique in an AWGN environment and without any knowledge of the signal structure is the energy detector. If we do assume some knowledge of the signal structure, then we can achieve a better performance.

Most of the wireless technologies in operation include the transmission of some sort of pilot sequence, to allow channel estimation, to beacon its presence to other terminals and to give a synchronization reference for subsequent messages. Secondary systems can exploit pilot signals in order to detect the presence of transmissions of primary systems in their vicinity.

If a pilot signal is known, then the matched filter signal detector achieves the optimal 
detection performance in AWGN channel, as shown by Tandra and Sahai in [17], as it maximizes the SNR.

Let us assume that:

- the signal detector knows the pilot sequence $x(k)$, the bandwidth and the center frequency in which it will be transmitted;

- the pilot sequence is always appended to each primary system's transmission (uplink or downlink);

- and the signal detector can always receive coherently.

Then, if $y(k)$ is a sequence of received samples at instant $k \in\{1,2, \ldots, N\}$ at the signal detector, the decision rule can be stated as [34]:

$$
\text { decide for }\left\{\begin{array}{ll}
H_{0}, & \text { if } \hat{\mathcal{S}}<\nu \\
H_{1}, & \text { if } \hat{\mathcal{S}} \geq \nu
\end{array},\right.
$$

where

$$
\hat{\mathcal{S}}=\sum_{k=1}^{N} y(k) x(k)^{\star}
$$

is the decision criterion, $\nu$ is the threshold to be compared and $x(k)^{\star}$ is the transpose conjugate of the pilot sequence.

Here the threshold $\nu$ is not the noise variance as it was for the energy detector. The hypothesis decision is simplified as the matched filter maximizes the power of $\mathcal{S}$ as seen in Eq. 1.7. This means it performs well even in a low SNR regime.

The matched filter has some drawbacks. A cognitive spectrum sensor might not know which networks are in operation in the environment at a given moment. Therefore it may 
not know which sets of pilots to look for. One must remember that if it tries to match an incorrect pilot, it will sense an empty medium and will incorrectly conclude that the medium is free. Second, the matched filter requires that every medium access be "signed" by a pilot transmission, but this is not the case in general. Furthermore, pilot sequences are only transmitted in the downlink direction. This leaves the uplink transmissions uncovered. Third, the matched filter requires coherent reception, which is generally hard to achieve in practice.

\section{Characterization of the Matched Filter}

Signal detection using the matched filter was studied in 2006 by Cabric, et. al. in [34]. They show that $\hat{\mathcal{S}}$ is Gaussian:

$$
\hat{\mathcal{S}} \sim \begin{cases}\mathcal{N}\left(0, \sigma_{n}^{2} \varepsilon\right), & H_{0} \\ \mathcal{N}\left(\varepsilon, \sigma_{n}^{2} \varepsilon\right), & H_{1}\end{cases}
$$

where $\sigma_{n}^{2}$ is the variance of the noise and

$$
\varepsilon=\sum_{k=1}^{N} x(k)^{2} .
$$

Based on this information, the probabilities of false alarm $P_{f}$ and detection $P_{d}$ are:

$$
P_{f}=Q\left(\frac{\hat{\mathcal{S}}}{\sqrt{\varepsilon \sigma_{n}^{2}}}\right)
$$

and

$$
P_{d}=Q\left(\frac{\hat{\mathcal{S}}-\varepsilon}{\sqrt{\varepsilon \sigma_{n}^{2}}}\right) .
$$




\subsubsection{Cyclostationary Feature Detection}

As we have seen, although it performs well, even in the low SNR regime, the matched filter requires a good knowledge of the signal structure, which secondary terminals may not have. The natural question to ask is whether we can still be able to perform spectrum sensing with a limited knowledge of the signal structure, perhaps based on a characteristic that is common to most known transmitted signals. In the following we show that it is indeed possible.

The cyclostationary feature detector relies on the fact that most signals exhibit periodic features, present in pilots, cyclic prefixes, modulations, carriers and other repetitive characteristics $[25,26,27,28,29,30]$. Since the noise is not periodic, the signal can be successfully detected.

The works by Gardner [25] in 1991 and Enserink et. al. [26] in 1995 have studied this signal detection scheme in detail. The work of Enserink et. al. follows the same line of the one by Gardner, in which the cyclostationary feature detector is based on the magnitudesquared of the spectral coherence, which for any random process $X$ is given by;

$$
\left|\rho_{X}^{\alpha}(f)\right|=\frac{\left|S_{X}^{\alpha}(f)\right|^{2}}{\left[<S_{X}>\left(f+\frac{\alpha}{2}\right)<S_{X}>\left(f-\frac{\alpha}{2}\right)\right]^{\frac{1}{2}}},
$$

where $S_{X}$ is the spectral correlation density function, $\alpha$ is the cyclic frequency and $f$ is the spectral frequency.

In the specific case of the cyclostationary feature detector, substituting $\rho_{X}^{\alpha}(f)$ by $\hat{\rho}_{X}^{\alpha}(f)$ and $S_{X}$ by $\hat{S}_{X}$, which are the estimated versions of the same quantities, we have the decision metric:

$$
\hat{\mathcal{M}}=\left|\hat{\rho}_{X}^{\alpha}(f)\right|=\frac{\left|S_{X}^{\alpha}(f)\right|^{2}}{<\hat{S}_{X}>\left(f+\frac{\alpha}{2}\right)<\hat{S}_{X}>\left(f-\frac{\alpha}{2}\right)},
$$

which goes into the decision statistic, given by 


$$
\text { decide for }\left\{\begin{array}{ll}
H_{0}, & \text { if } \hat{\mathcal{M}}<\nu \\
H_{1}, & \text { if } \hat{\mathcal{M}} \geq \nu
\end{array}\right. \text {, }
$$

A newer work focuses on cyclostationary feature detector for cognitive radio networks [30], called multi-cycles detector. In this work, a cyclostationarity detector scheme is employed on non-filtered signals, such as OFDM, to detect the cyclic frequency and its harmonics. Finally, it is thought that the cyclostationary feature detector is the most promising signal detection technique as it combines good performance with low requirements on the knowledge of the signal structure [27].

\subsection{Cooperative Sensing Techniques}

While for simple AWGN channels most classical approaches perform well, as we have seen, in the case of fading these techniques are not able to provide satisfactory results due to their inherent limitations and to the hidden node problem. To this end, several works $[32,33,34,42,44,35,36,37,41]$ have looked into the case in which cooperation is employed in sensing the spectrum.

Consider the scenario depicted in figure 1.3, in which primary users (in white) communicate with their dedicated (primary) base station. Secondary receivers $\left\{R X_{1}, R X_{2}, R X_{3}, \ldots, R X_{K}\right\}$ cooperatively sense the channel to identify a white space and exploit the medium. The main idea of the cooperative sensing techniques is that each receiver $R X_{i}$ can individually measure the channel and interact on their findings to decide if the medium is available. The main drive behind this idea is that each secondary receiver will have a different perception of the spectrum, as its channel to the receiver will be different from the other secondary receivers, thus decreasing the chances of interfering with hidden nodes.

Alternative scenarios exist, ,

We will concentrate on the one pictured in Fig. 1.3, although all sensing techniques 


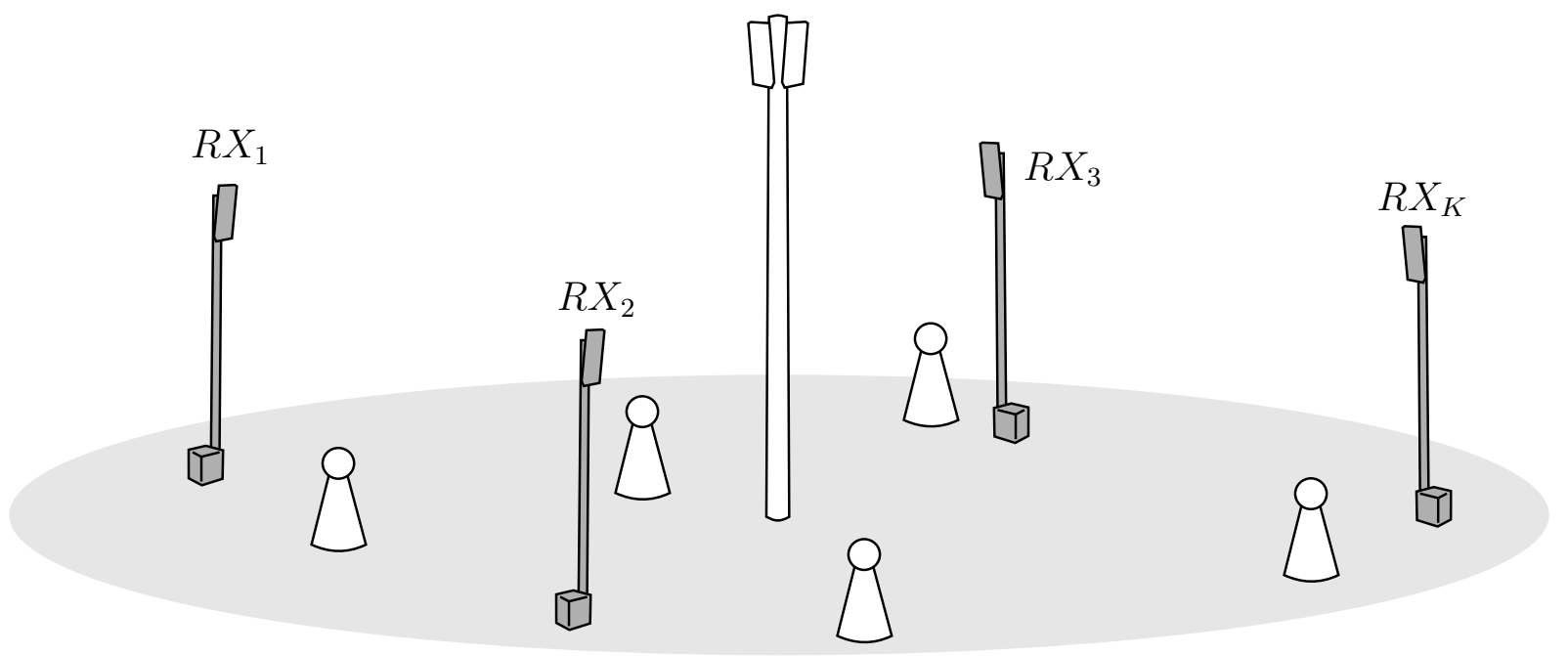

Figure 1.3: Cooperative sensing scenario

presented herein can be also applied to scenarios such as the one in which a deployment of a secondary network exclusively for spectrum sensing is considered, as shown in [31].

The cooperative spectrum sensing can be [44]:

- Centralized, in which a central entity gathers all information from all secondary receivers to make a decision about the medium status, which is then transmitted back to the receivers;

- Distributed, in which the receivers share their information in order to make their own decision.

In both of these situations, the cooperative spectrum sensing is plagued with one problem: how to report or distribute the measures in a resource constrained network. In fact, if these measurements are the basis for deciding whether a transmission can be made or not, then it does not make any sense to propagate the measurements before the decision is made. To overcome the problem, one could create a dedicated channel for signaling (such as in [?]) or use unregulated band (such as ISM). Other works [32, 33, 34, 42, 44, 35, 36] try to restrict the reporting to the minimum possible (often one bit) to ease the process of distributing this information. Finally, [37] considers a hierarchically structured secondary network, in which the secondary spectrum sensors are the secondary base stations, distributed over the sensing 
area. These base stations would make use of a backbone with enough bandwidth to distribute the measurements among themselves, irrespective of being a single bit or the actual acquired data. Then, during a white space, the terminals are be signaled to transmit. Nevertheless, secondary base stations, as opposed to secondary terminals, have more processing power and fewer power constraints so they can better perform the spectrum sensing task. It should be noted both of these approaches has its own target application; neither can be considered the best approach in every case.

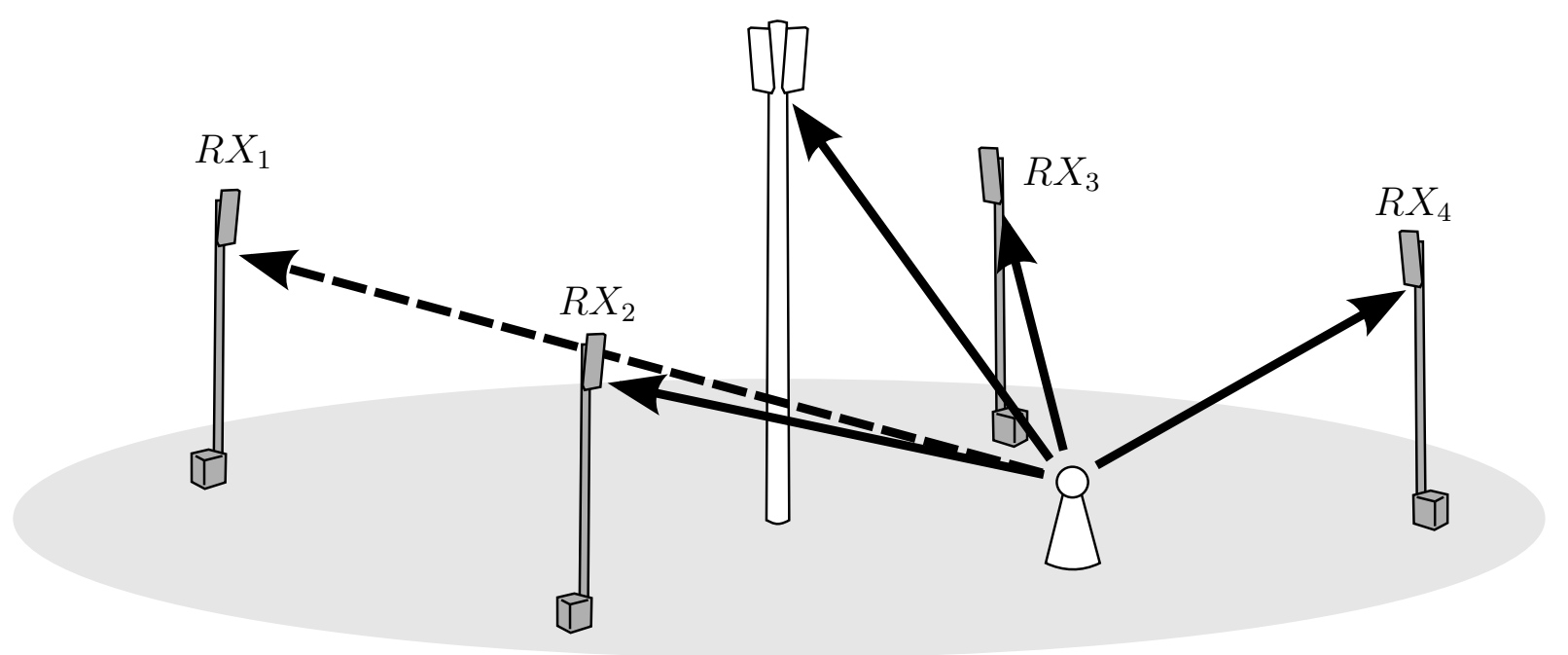

Figure 1.4: Cooperative Sensing Scenario

Another problem of cooperative spectrum sensing is the decision of which secondary receivers are to be considered reliable. Let us consider the situation picture in Fig. 1.4, in which one primary terminal is transmitting data in the uplink channel (with low power) towards its primary base station. Several spectrum sensors $\left\{R X_{1}, R X_{2}, R X_{3}, R X_{4}\right\}$ are monitoring the medium detect its state. In this example, $\left\{R X_{2}, R X_{3}, R X_{4}\right\}$ are in range of the transmitter and can correctly sense its ongoing transmission, but $R X_{1}$ is not ${ }^{1}$. Thus, when the measures of all of the sensors are gathered, how does one select the individual receivers that are performing a reliable measurement? Without knowing the position of the primary transmitter and the channels between secondary receivers and the primary transmitter, this is a complicated task. The work by Mishra et. al. [42] looks further in to the performance impacts that the lack of reliability yields. Some works [27, 43] discuss about a weighting scheme to give different scales to different secondary receivers base on

\footnotetext{
${ }^{1}$ This would also apply to the case where $R X_{1}$ is shadowed or in a deep multi-path fading
} 
their channel. Other works $[32,33,34,35,36]$ propose a voting scheme in order to make a trustworthy decision, even with the presence of doubtful measurements.

In the remainder of this section we will explore some of the state-of-the-art cooperative sensing techniques.

\subsubsection{Voting Based Sensing}

We saw in Sec. 1.3.1 that, in the low SNR regime, the energy detector is highly vulnerable to fading and fluctuations in the level of the noise power. What if, instead of employing the energy detector at one location, we could do the same thing in other locations as well? It is expected that among several secondary receivers, even though some will suffer from fading or imprecisions due to the choice of the threshold, some will be able to correctly sense the medium. This is the main idea behind the collaborative spectrum sensing based on voting, studied in a number of works $[32,33,34,35,36]$.

In the voting spectrum sensing each secondary receiver $R X_{i}$ uses spectrum sensing to form its own decision, as presented in Sec. 1.3.1. Consider the vector of all responses $\mathbf{r}$ such that

$$
\mathbf{r}=\left[\begin{array}{lllll}
r_{1} & r_{2} & r_{3} & \ldots & r_{K}
\end{array}\right]
$$

where $r_{i} \in\{1,0\}$ is the binary response for each sensor $i$. After all measurements are gathered the voting procedure takes place [32, 33, 34]:

$$
\text { decide for }\left\{\begin{array}{ll}
H_{0}, & \text { if } \mathcal{V}=0 \\
H_{1}, & \text { if } \mathcal{V} \geq 0
\end{array}\right. \text {, }
$$

where 


$$
\mathcal{V}=\sum_{k=1}^{K} r_{k}
$$

Briefly, the voting schemes selects $H_{1}$ if at least one of the secondary receivers decides for $H_{1}$, which is known as the OR rule. Although this may seem too pessimistic, as it will favor false alarms, according to $[32,33,34]$, this already gives improvements over the simple energy detection case even for two users. This is reasonable if we remark that with a high number of sensors, higher the probability of reliable spectrum sensing among secondary receivers. The probabilities of detection and false alarm for the cooperative approach are

$$
Q_{f}=1-\left(1-P_{f}\right)^{K}
$$

and

$$
Q_{d}=1-\left(1-P_{d}\right)^{K}
$$

respectively.

The work by Sun et. al. [35] revisits this scheme, to estimate the reliability of each node. In this scheme only the nodes with reliable sensing are allowed to report their detection. The reliability measure is based on how close the energy of $y(k)$ is to $\nu$, as seen in Fig. 1.5.

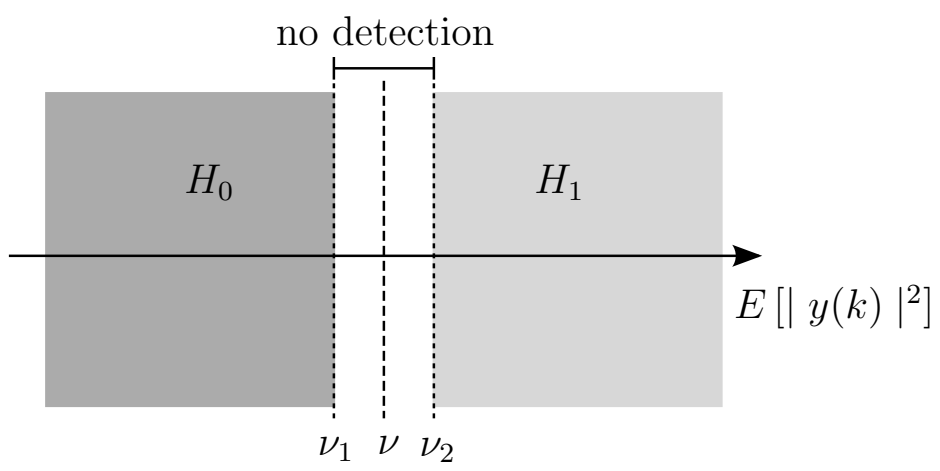

Figure 1.5: Reliability decision scheme. 
This work defines two new thresholds, $\nu_{1}$ and $\nu_{2}$, that are used to define a "no decision" region. Thus the decision rule can be stated as

$$
\text { decide for }\left\{\begin{array}{l}
H_{0}, \quad \text { if } 0 \leq \mathcal{E} \leq \nu_{1} \\
H_{1}, \quad \text { if } \mathcal{E} \geq \nu_{2}
\end{array} .\right.
$$

If $\mathcal{E}$ falls in $\left(\nu_{1}, \nu_{2}\right)$, then the secondary receiver decides not to report. This way the overall decision, based on the OR rule, concentrates on the reports of $M$ users with a reliable detection out of $K$ total users. The results from this work suggest an increased performance over the conventional case, where no reliability information is used.

Another work from Sun et. al. [36] propose a cluster based spectrum sensing. In this work, a cluster is a grouping of secondary receivers which are spatially close. In each cluster, one receiver, called the cluster head, is elected to do the local decision and the reporting to the central decision entity. There the final decision takes place.

\subsubsection{Correlator Based Sensing}

Another possibility is to gather all received samples at a central entity that will take the decision instead of leaving the decision of the medium availability to the secondary receivers. With an overall view of the situation, the central entity can decide how to better manage the measurements for the decision taking task. The schemes presented in this and the next sections all involve such a central entity.

Let us, for simplicity sake, suppose that all secondary receivers $\left\{R X_{1}, R X_{2}, R X_{3}, \ldots, R X_{K}\right\}$, shown in figure 1.3 are within the range of a certain primary transmitter. Then, considering a flat-faded environment, we have:

$$
y_{i}(k)= \begin{cases}n_{i}(k): & H_{0} \\ h_{i}(k) s(k)+n_{i}(k): & H_{1}\end{cases}
$$


where the subscript $i$ means that each value is to be taken for each user $i$.

We can see that for the $H_{0}$ hypothesis, all $y_{i}(k)$ are independent since they are only composed of AWGN noise. On the other hand, in the $H_{1}$ hypothesis, all $y_{i}(k)$ are composed of not only the noise, but also the signal component $s(k)$ modulated by the channel $h_{i}(k)$. As we know, the signal is common for all users, since it is broadcast by the primary transmitter. We can exploit this fact to detect the presence of transmitted signals by focusing on the correlation between received signals from secondary receivers.

This correlation is calculated via the cyclic convolution, defined as:

$$
R_{(i j)}(k)=\sum_{k=1}^{N} y_{i}(a) y_{j}((k-a) \bmod N)
$$

where $i$ and $j$ are the indices of any two secondary receivers.

In his scheme the decision rule is given by:

$$
\text { decide for }\left\{\begin{array}{ll}
H_{0}, & \text { if } \mathcal{L}<\nu \\
H_{1}, & \text { if } \mathcal{L} \geq \nu
\end{array},\right.
$$

where $\mathcal{L}$ is the decision statistic calculated as

$$
\mathcal{L}=\max _{(i, j) \in \mathcal{B}} \max _{k}\left(R_{(i j)}\right),
$$

where $R_{(i j)}$ is the pairwise cyclic convolution for all permutation of secondary receivers, $\mathcal{B}=\{(x, y) \in \mathcal{A} \times \mathcal{A} \mid y \geq x+1\}$ and $\mathcal{A}=\{1,2, \ldots, N\}$. Note that differently from the matched filter, this scheme does not require coherent reception, as it looks for the highest correlation between any two pairs of sensors. Nevertheless in the case of coherent reception, we could rewrite Eq. 1.15 as 


$$
\mathcal{L}=\sum_{k=1}^{N}\left(R_{(i j)}\right), \forall(i, j) \mid i \neq j
$$

which would effectively maximize the SNR.

As far as the authors know, this spectrum sensing scheme has not yet been studied in the literature and thus its performance is not known. It would likely suffer from the same problem as the matched filter, namely, the challenge of correctly choosing $\nu$. The main limitation of this scheme would be its necessity to report all the measurements, which would require an infrastructure with a very high bandwidth dedicated for the task.

\subsubsection{Eigenvalue Based Sensing}

Eigenvalue based sensing is another technique for cooperative sensing, introduced by Cardoso et. al. [37] and Zeng et. al. [41], based on evaluating the eigenvalues of a matrix formed by the samples collected by multiple sensors in relation to the Marchenko-Pastur law. Herein, we will explore the approach as was presented in [37] since the approach in [41] is very similar.

In order to better understand how this spectrum sensing procedure works, we start with the following assumption:

- The $K$ base stations in the secondary system share information between them. This can be performed by transmission over a wired high speed backbone.

- The base stations are analyzing the same portion of the spectrum.

Let us consider the following $K \times N$ matrix consisting of the samples received by all the $K$ secondary receivers $R X_{i}$ : 


$$
\mathbf{Y}=\left[\begin{array}{cccc}
y_{1}(1) & y_{1}(2) & \cdots & y_{1}(N) \\
y_{2}(1) & y_{2}(2) & \cdots & y_{2}(N) \\
y_{3}(1) & y_{3}(2) & \cdots & y_{3}(N) \\
\vdots & \vdots & & \vdots \\
y_{K}(1) & y_{K}(2) & \cdots & y_{K}(N)
\end{array}\right]
$$

Then, the objective of the eigenvalue based approach is to perform a test of independence of the signals received at $R X_{i}$. As said before, in the $H_{1}$ case, all the received samples are expected to be correlated, whereas when in the $H_{0}$ case, the samples are decorrelated. Hence, in this case, for a fixed $K$ and $N \rightarrow \infty$, under the $H_{0}$ assumption the sample covariance matrix $\frac{1}{N} \mathbf{Y} \mathbf{Y}^{H}$ converges to $\sigma^{2} \mathbf{I}$. However, in practice, $N$ can be of the same order of magnitude than $K$ and therefore one can not infer directly $\frac{1}{N} \mathbf{Y} \mathbf{Y}^{H}$ independence of the samples. This can be formalized using tools from random matrix theory [38]. In the case where the entries of $\mathbf{Y}$ are independent (irrespective of the specific probability distribution, which corresponds to $H_{0}$ ) we can use the following result from asymptotic random matrix theory [38]:

Theorem. Consider an $K \times N$ matrix $\mathbf{W}$ whose entries are independent zero-mean complex (or real) random variables with variance $\frac{\sigma^{2}}{N}$ and fourth moments of order $O\left(\frac{1}{N^{2}}\right)$. As $K, N \rightarrow \infty$ with $\frac{K}{N} \rightarrow \alpha$, the empirical distribution of $\mathbf{W} \mathbf{W}^{H}$ converges almost surely to a nonrandom limiting distribution with density

$$
f(x)=\left(1-\frac{1}{\alpha}\right)^{+} \delta(x)+\frac{\sqrt{(x-a)^{+}(b-x)^{+}}}{2 \pi \alpha x}
$$

where

$$
a=\sigma^{2}(1-\sqrt{\alpha})^{2} \quad \text { and } \quad b=\sigma^{2}(1+\sqrt{\alpha})^{2},
$$

which is known as the Marchenko-Pastur law. 
Interestingly, under the $H_{0}$ hypothesis, the support of the eigenvalues of the sample covariance matrix (in Figure 1.6, denoted by $\check{\mathrm{MP}}$ ) is finite. The Marchenko-Pastur law thus serves as a theoretical prediction under the assumption that matrix is "all noise". Deviations from this theoretical limit in the eigenvalue distribution should indicate nonnoisy components.

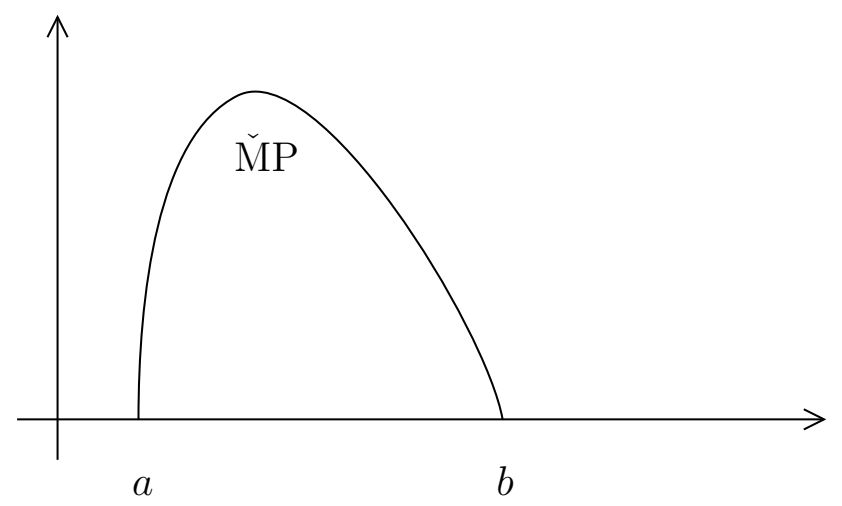

Figure 1.6: The Marchenko-Pastur support ( $H_{0}$ hypothesis).

In the case in which a signal is present $\left(H_{1}\right), \mathbf{Y}$ can be rewritten as

$$
\mathbf{Y}=\left[\begin{array}{cccc}
h_{1} & \sigma & & 0 \\
\vdots & & \ddots & \\
h_{K} & 0 & & \sigma
\end{array}\right]\left[\begin{array}{ccc}
s(1) & \cdots & s(N) \\
z_{1}(1) & \cdots & z_{1}(N) \\
\vdots & & \vdots \\
z_{K}(1) & \cdots & z_{K}(N)
\end{array}\right]
$$

where $s(k)$ and $z_{i}(k)=\sigma n_{i}(k)$ are, respectively, the independent signal and noise with unit variance at instant $k$ and secondary receiver $i$. Let us denote by $\mathbf{T}$ the matrix:

$$
\mathbf{T}=\left[\begin{array}{cccc}
h_{1} & \sigma & & 0 \\
\vdots & & \ddots & \\
h_{K} & 0 & & \sigma
\end{array}\right]
$$

$\mathbf{T} \mathbf{T}^{H}$ clearly has one eigenvalue equal to $\lambda_{1}=\sum\left|h_{i}\right|^{2}+\sigma^{2}$ and all the rest equal to $\sigma^{2}$. The behavior of the eigenvalues of $\frac{1}{N} \mathbf{Y} \mathbf{Y}^{H}$ is related to the study of the eigenvalues of large 
sample covariance matrices of spiked population models [39]. Here, the SNR $\xi$ is defined as

$$
\xi=\frac{\sum\left|h_{i}\right|^{2}}{\sigma^{2}}
$$

The works by Baik et al.[39, 40] have shown that, when

$$
\frac{K}{N}<1 \quad \text { and } \quad \xi>\sqrt{\frac{K}{N}}
$$

(which are assumptions that are clearly met when the number of samples $N$ are sufficiently high), the maximum eigenvalue of $\frac{1}{N} \mathbf{Y} \mathbf{Y}^{H}$ converges almost surely to

$$
b^{\prime}=\left(\sum\left|h_{i}\right|^{2}+\sigma^{2}\right)\left(1+\frac{\alpha}{\xi}\right),
$$

which is greater than the value of $b=\sigma^{2}(1+\sqrt{\alpha})^{2}$ seen in the $H_{0}$ case.

Therefore, whenever the distribution of the eigenvalues of the matrix $\frac{1}{N} \mathbf{Y} \mathbf{Y}^{H}$ departs from the Marchenko-Pastur law, as seen in Fig. 1.7, the detector decides that the signal is present. Hence, we apply this feature from a spectrum sensing point of view.

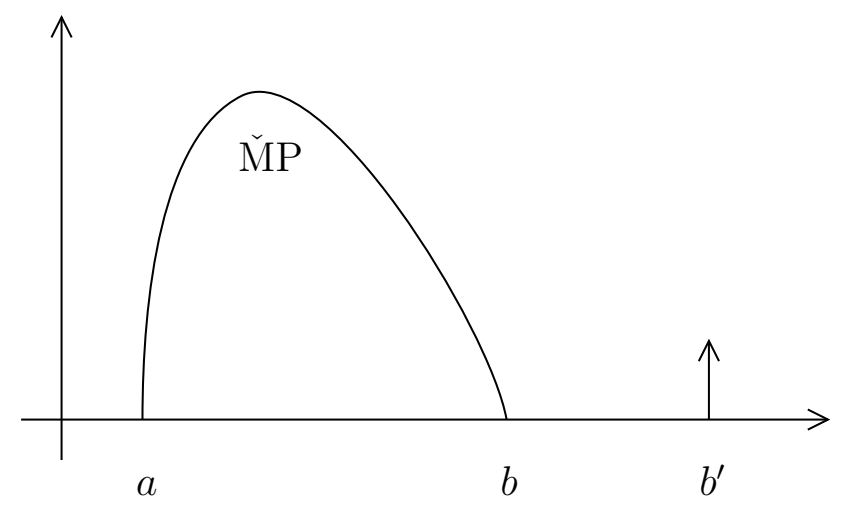

Figure 1.7: The Marchenko-Pastur support plus a signal component.

Considering $\lambda_{i}$ be the eigenvalues of $\frac{1}{N} \mathbf{Y} \mathbf{Y}^{H}$ and letting $G=[a, b]$, the cooperative sensing scheme works in two possible ways: 
Noise distribution unknown, variance known

In this case, the decision criteria used is

$$
\text { decide for }\left\{\begin{array}{lc}
H_{0}: & \text { if } \lambda_{i} \in G \\
H_{1}: & \text { otherwise. }
\end{array}\right.
$$

\section{Both noise distribution and variance unknown}

The ratio of the maximum and the minimum eigenvalues in the $H_{0}$ hypothesis case does not depend on the noise variance and thus serves well as a criteria independent from the noise

$$
\text { decide for }\left\{\begin{array}{lc}
H_{0}: & \text { if } \frac{\lambda_{\max }}{\lambda_{\min }} \leq \frac{(1+\sqrt{\alpha})^{2}}{(1-\sqrt{\alpha})^{2}} \\
H_{1}: & \text { otherwise. }
\end{array}\right.
$$

It should be noted that, in this case, one needs to still take a sufficiently high number of samples $N$ such that the conditions in Eq. (1.16) are met. In other words, the number of samples scales quadratically with the inverse of the signal to noise ratio. Note moreover that the test under $H_{1}$ hypothesis also provides a good estimator of the SNR $\rho$. Indeed, the ratio of largest eigenvalue $\left(b^{\prime}\right)$ and smallest $(a)$ of $\frac{1}{N} \mathbf{Y} \mathbf{Y}^{H}$ is related solely to $\rho$ and $\alpha$ :

$$
\frac{b^{\prime}}{a}=\frac{(\rho+1)\left(1+\frac{\alpha}{\rho}\right)}{(1-\sqrt{\alpha})^{2}}
$$

\subsection{Conclusions and Open Issues}

In this chapter, the state-of-the-art of spectrum sensing techniques for cognitive radio were covered. We presented not only the classical techniques, inspired by the signal detection approaches developed for radar systems, but also some newly developed ones, carefully tai- 
lored for the cognitive radio network scenario. Furthermore, we presented their operation, characteristics, advantages, and limitations.

In spite of the popularity of spectrum sensing as a study subject in cognitive radio and cognitive radio networks, there are still some open subjects in this area. Generally, the study has tackled the sensing techniques themselves but little work has considered the systemic point of view, implementation issues and complexity of techniques concerning the spectrum sensing. Some open issues can be enumerated:

- Adaptive spectrum sensing. The techniques for spectrum sensing studied so far consider well-behaved scenarios. For some of these techniques, it is quite clear that time-varying environments would greatly compromise their performance. Since cognitive radio networks will most likely operate in such environments, it is important that adaptive spectrum sensing techniques be devised.

- Cooperation between primary and secondary systems. Is spectrum sensing the best way to find out the medium availability? In some scenarios, maybe not. Of course the cognitive radio network idea is to incur in the minimum possible changes to the primary systems, but it may be that in some specific scenarios, by sharing some information to spectrum brokers, primary systems will benefit from less, or even zero, interference from secondary systems.

- Cooperative sensing. It is clear that the cooperative sensing may be the best option for spectrum sensing in many faded environments. If this is so, how can we overcome the issues related to sensing time, error-prone metric distribution and reliability that still plague the available tchniques? What is the holy grail of cooperative sensing? Is there one?

- Complexity and implementation issues. One of the main limitations of the cognitive radios, and hence of spectrum sensing, is the physical limitation of the hardware and radio frequency $(\mathrm{RF})$ components required. Today, no one knows how to create these cognitive radio transceivers in production scale, with a small package and consuming low power. Another open question is how to focus the sensing energy. While wide-band sensing would give a faster and clearer overall picture of the spectrum, it 
would provide a very rough perspective, since the sensing energy is distributed over a large spectrum. On the other hand, sweeping the spectrum with narrowband sensing concentrates the sensing energy, yielding better precision, but that might take too long to give the overall picture. Furthermore, since it is envisioned that sensing will be done by terminals, how do all sensing techniques compare in terms of implementation complexity, energy usage and processing power?

- Cognitive Pilot Channel. One interesting new concept in the cognitive radio network arena that requires further study is the cognitive pilot channel (CPC). The CPC is a specific frequency channel reserved for the diffusion of cognitive radio related information, such as current frequency band allocation. The main advantages of the CPC is the centralization of information, faster cognitive radio connection to target networks and the relaxation on the requirements of sensing. CPC transmitters can gather frequency band availability information through explicit notification by neighboring networks and spectrum sensing relieving the power and processing limited nodes from this task. Nevertheless, the CPC is very useful for long range communications, whereas for personal area networks it might be meaningless and even misleading. It will also depend up to a certain extent on the good will of neighboring network operators to offer such information.

\section{References}

[1] M.I. Skolnik. Introduction to Radar Systems. McGraw-Hill Singapore, 1980.

[2] J. Mitola. Cognitive Radio An Integrated Agent Architecture for Software Defined Radio. $\mathrm{PhD}$ thesis, Royal Institute of Technology (KTH), May 2000.

[3] S. Haykin. Cognitive radio: brain-empowered wireless communications. Selected Areas in Communications, IEEE Journal on, 23(2):201-220, 2005.

[4] I. F. Akyildiz, W. Y. Lee, M.C. Vuran, and S. Mohanty. NeXt generation/dynamic spectrum access/cognitive radio wireless networks: a survey. Comput. Networks, 50(13):2127-2159, 2006.

[5] Spectrum Efficiency Working Group. Report of the Spectrum Efficiency Working Group. 
Technical report, FCC, November 2002.

[6] J. Palicot and C. Roland. A new concept for wireless reconfigurable receivers. Communications Magazine, IEEE, volume 41, number 7, pages 124-132, 2003.

[7] A. Fehske, J. Gaeddert and J.H.. A new approach to signal classification using spectral correlation and neural networks. Proc. IEEE Int. Symp. New Frontiers Dynamic Spectr. Access Networks, volume 1, pages 144-150, 2005.

[8] R. Hachemani, J. Palicot and C. Moy. A new standard recognition sensor for cognitive radio terminal. USIPCO 200\%, 3-7 September, Poznan, Poland, 2007.

[9] A. Bouzegzi, P. Jallon and P.Ciblat. A second order statistics based algorithm for blind recognition of OFDM based systems In Proc. Globecom 2008, 2008.

[10] A. Sahai and D. Cabric. Spectrum sensing: fundamental limits and practical challenges. Tutorial presented at the 1st IEEE Conference on Dynamic Spectrum Management (DySPAN05), 2005.

[11] Z. Tian and G.B. Giannakis. A Wavelet Approach to Wideband Spectrum Sensing for Cognitive Radios. Cognitive Radio Oriented Wireless Networks and Communications, 2006. 1st International Conference on, pages 1-5, 2006.

[12] Y. Hur, J. Park, W. Woo, K. Lim, C.H. Lee, H.S. Kim, J. Laskar. A wideband analog multi-resolution spectrum sensing (MRSS) technique for cognitive radio (CR) systems. Circuits and Systems, 2006. ISCAS 2006. Proceedings. 2006 IEEE International Symposium on, pages 4, 2006.

[13] Z. Quan, S. Cui, A.H. Sayed, V. Poor, Wideband spectrum sensing in cognitive radio networks. arXiv:0802.4130, 2008.

[14] HL. Vantrees, Detection, Estimation and Modulation Theory. Wiley, vol. 1, 1968.

[15] V. Poor, An Introduction to Signal Detection and Estimation. Springer, 1994.

[16] H. Urkowitz. Energy detection of unknown deterministic signals. Proceedings of the IEEE, 55:523-531, 1967.

[17] R. Tandra and A. Sahai. Fundamental limits on detection in low SNR under noise uncertainty. International Conference on Wireless Networks, 2005.

[18] J.G. Proakis, and M. Salehi. Digital Communications, McGraw-Hill New York, 1995.

[19] S.S. Haykin. Communication systems, Wiley New York, 2001.

[20] C.L. Fullmer and JJ Garcia-Luna-Aceves. Solutions to hidden terminal problems in 
wireless networks. Proceedings of the ACM SIGCOMM'97 conference on Applications, technologies, architectures, and protocols for computer communication, pages 39-49, 1997.

[21] V. I. Kostylev. Energy detection of a signal with random amplitude. 2002.

[22] F. F. Digham, M. S. Alouini, and M. K. Simon. On the energy detection of unknown signals over fading channels. 2003.

[23] A. Sahai, N. Hoven, and R. Tandra. Some fundamental limits on cognitive radio. Allerton Conference on Communication, Control, and Computing, 2003.

[24] D. Cabric, A. Tkachenko and R.W. Brodersen. Spectrum Sensing Measurements of Pilot, Energy, and Collaborative Detection, In Proc. of IEEE Military Communications Conference October, 2006.

[25] W.A. Gardner. Exploitation of spectral redundancy in cyclostationary signals. Signal Processing Magazine, IEEE, volume 8, number 2, pages 14-36, 1991.

[26] S. Enserink, and D. Cochran. A cyclostationary feature detector. In Signals, Systems and Computers, 1994. 1994 Conference Record of the Twenty-Eighth Asilomar Conference on, volume 2, 1995.

[27] D. Cabric, S. M. Mishra, and R. W. Brodersen. Implementation issues in spectrum sensing for cognitive radios. In Signals, Systems and Computers, 2004. Conference Record of the Thirty-Eighth Asilomar Conference on, volume 1, pages 772-776 Vol.1, 2004.

[28] A. Fehske, J. Gaeddert, and JH Reed. A new approach to signal classification using spectral correlation and neural networks. In Proc. IEEE Int. Symp. New Frontiers Dynamic Spectr. Access Networks, 1:144-150.

[29] H. Tang. Some physical layer issues of wide-band cognitive radio systems. Proc. IEEE Int. Symp. New Frontiers Dynamic Spectr. Access Networks, 1:151-159.

[30] M. Ghozzi, M. Dohler, F. Marx and J. Palicot. Cognitive radio: methods for the detection of free bands. Comptes Rendus Physique, Elsevier, volume 7, pages 794-804, September 2006, 2006.

[31] S.N. Shankar, C. Cordeiro and K. Challapali. SpectrumAgile Radios: Utilization and Sensing Architectures. First IEEE International Symposium on New Frontiers in Dynamic Spectrum Access Networks, pages 160-169, 2005. 
[32] A. Ghasemi and E. S. Sousa. Collaborative spectrum sensing for opportunistic access in fading environments. New Frontiers in Dynamic Spectrum Access Networks, 2005. DySPAN 2005. 2005 First IEEE International Symposium on, pages 131-136, 2005.

[33] A. Ghasemi and E. S. Sousa. Spectrum sensing in cognitive radio networks: the cooperation-processing tradeoff. Wireless Communications and Mobile Computing WILEY, 200\%., pages 1049-1060, 2007.

[34] D. Cabric, A. Tkachenko and R.W. Brodersen. Spectrum Sensing Measurements of Pilot, Energy, and Collaborative Detection. In Proc. of IEEE Military Communications Conference, Oct, 2006.

[35] C. Sun, W. Zhang, and K.B. Letaief. Cooperative Spectrum Sensing for Cognitive Radios under Bandwidth Constraints. In Wireless Communications and Networking Conference, 200\%. WCNC 200\%. IEEE, pages 1-5, 2007.

[36] C. Sun, W. Zhang, and K.B. Letaief. Cluster-Based Cooperative Spectrum Sensing in Cognitive Radio Systems. In Communications, 200\%. ICC'0\%. IEEE International Conference on, pages 2511-2515, 2007.

[37] L. S. Cardoso, M. Debbah, P. Bianchi, and J. Najim. Cooperative Spectrum Sensing Using Random Matrix Theory. In International Symposium on Wireless Pervasive Computing ISWPC 2008, 2008.

[38] V. A. Marchenko and L. A. Pastur. Distributions of eigenvalues for some sets of random matrices. Math USSR-Sbornik, 1:457-483, 1967.

[39] J. Baik, G. B. Arous, and S. Peche. Phase transition of the largest eigenvalue for nonnull complex sample covariance matrices. Ann. Probab, 33(5):1643-1697, 2005.

[40] J. Baik and J. W. Silverstein. Eigenvalues of large sample covariance matrices of spiked population models. J. Multivar. Anal., 97(6):1382-1408, 2006.

[41] Y. Zeng and Y. C. Liang. Eigenvalue base Spectrum Sensing Algorithms for Cognitive Radio arXiV:0804.2960, 2008.

[42] S. M. Mishra, A. Sahai, and R. W. Brodersen. Cooperative sensing among cognitive radios. In Communications, 2006. ICC '06. IEEE International Conference on, volume 4, pages $1658-1663,2006$.

[43] X. Huang, N. Han, G. Zheng, S. Sohn, and J. Kim. Weighted-Collaborative Spectrum Sensing in Cognitive Radio. In Communications and Networking in China, $200 \%$. 
CHINACOM'0\%. Second International Conference on, pages 110-114, 2007.

[44] G. Ganesan and Y. Li. Cooperative spectrum sensing in cognitive radio networks. In New Frontiers in Dynamic Spectrum Access Networks, 2005. DySPAN 2005. 2005 First IEEE International Symposium on, pages 137-143, 2005. 